\title{
Single-cell metabolic imaging reveals a RhoA- triggered glycolytic burst in motile endothelial cells
}

\section{David Wu}

University of Chicago https://orcid.org/0000-0003-3162-3238

\section{Devin Harrison}

University of Chicago

\section{Teodora Szasz}

University of Chicago

\section{Chih-Fan Yeh}

University of Chicago

\section{Tzu-Pin Shentu}

University of Chicago

\section{Angelo Meliton}

University of Chicago

Ru-Ting Huang

University of Chicago

\section{Zhengjie Zhou}

University of Chicago

Gökhan Mutlu

University of Chicago

Jun Huang ( $\square$ huangjun@uchicago.edu )

University of Chicago

Yun Fang ( $\nabla$ yfang1@medicine.bsd.uchicago.edu )

University of Chicago

\section{Research Article}

Keywords: single, cell, metabolism, thrombin, RhoA, contraction, subcellular

Posted Date: January 18th, 2021

DOI: https://doi.org/10.21203/rs.3.rs-149025/v1

License: (c) (i) This work is licensed under a Creative Commons Attribution 4.0 International License.

Read Full License 
Version of Record: A version of this preprint was published on May 24th, 2021. See the published version at https://doi.org/10.1038/s42255-021-00390-y. 


\section{Abstract}

Single-cell motility is spatially heterogeneous and driven by metabolic energy. Direct linking cell mobility to cell metabolism is technically challenging but biologically important. Here we implemented a singlecell metabolic imaging assay to measure glycolysis in individual endothelial cells using geneticallyencoded biosensors capable of deciphering metabolic heterogeneity with subcellular resolution. We observed that cellular glycolysis fuels endothelial activation, migration and contraction and that the high lactate production sites co-localize with active cytoskeletal remodeling within an endothelial cell. Mechanistically, we found RhoA induces endothelial glycolysis for the phosphorylation of cofilin and myosin light chain in order to reorganize the cytoskeleton and thus control cell mobility; RhoA activation triggers a glycolytic burst through the translocation of a glucose transporter SLC2A3/GLUT3 to fuel the cellular contractile machinery, as demonstrated across multiple endothelial types. Together, our results discovered that Rho-GTPase signaling coordinates energetic metabolism with cytoskeleton remodeling to regulate the motility of single endothelial cells.

\section{Introduction}

Endothelial cells exhibit motile behaviors in response to hemodynamics, during migration in angiogenesis, and during contractions in barrier failure; each of which involves drastic morphological changes that require energy production and consumption. Bulk measurements have revealed that 1) endothelial cells preferentially utilize glycolysis for fueling migration ${ }^{1}, 2$ ) endothelial cells are dynamically regulated by shear stress ${ }^{2-4}$, and 3 ) endothelial motilities are tightly regulated by the Rho family of small GTPases ${ }^{5,6}$. However, mechanistic coupling of motility to energy in typically heterogeneous cell populations requires simultaneous information at the single-cell level. Therefore, there is a critical need for developing single-cell metabolic measurements which can inform new mechanisms by linking cell motility phenotypes to energy production in the same cell. Furthermore, measuring energy production in spatially disparate subcellular structures of a single cell is expected to have mechanistic significance in cell biology since most cells dynamically regulate their metabolic programs as a result of nutrient availability, anabolic requirements, and energy demand.

By using single-cell imaging analysis, we were able to link glycolysis to motility in individual endothelial cells in order to understand spatially heterogeneous phenomena. This Förster resonance energy transfer (FRET) image-based approach uses deep learning ${ }^{7-10}$ to rapidly quantify the intracellular glycolytic metabolites including lactate, glucose, pyruvate, and ATP in single endothelial cells, thus linking metabolic states to key endothelial motile phenotypes including adaptation to shear stress, migration, and contraction. A strong correlation between morphology and glycolytic rate at the single-cell level was identified, which was further confirmed by the subcellular distribution of high glycolytic rates at sites of active actin turnover. Our single-cell metabolic measurements discovered that the activation of GTPase RhoA triggers a glycolytic burst essential for the endothelial contractile machinery. We further discovered 
that glucose transporter SLC2A3/GLUT3 controls the RhoA-induced endothelial glycolysis and subsequent contraction. These results highlight the significance of single-cell metabolic analyses in their ability to uncover a new paradigm of small GTPase-dependent coupling of energy production to cell mobility.

\section{Results}

\section{Development of a single-cell metabolic imaging method}

To study motile phenotypes of individual endothelial cells, we first devised a single-cell metabolism quantification method to monitor the lactate concentration and glycolytic rate of individual cells expressing a genetically encoded biosensor. Here we used Laconic, a Förster resonance energy transfer (FRET)-based lactate biosensor comprising of an mTFP/Venus FRET pair and a bacterial transcription factor LIdR ${ }^{11}$. LIdR binds lactate causing the FRET pair to change their intramolecular distance that leads to increased mTFP (acceptor)/Venus (donor) (or 1/FRET) signal. In this way, lactate concentrations are positively proportional to 1/FRET signals (Extended Data Fig. 1a). Laconic-expressing human endothelial cells were imaged, segmented using a semantic segmentation pipeline, and assembled into fluorescence traces under biochemical and/or biomechanical stimulations (Fig. 1a). This allowed us to monitor the terminal metabolite in glycolysis, lactate, in endothelial cells, thus directly revealing endothelial glycolytic reprogramming for energy production. A deep learning approach with U-nets ${ }^{10}$ using custom code in MATLAB rapidly and reliably performed pixel-level classification of images and segmentation of single cells with Boundary F1 score ${ }^{12}$ for identifying the cytoplasm $>90 \%$, and class-specific cytoplasm accuracy, precision, recall, and Dice score $>80 \%$, (Extended Data Fig. 1b, Extended Data Table 1). Singlecell fluorescence following segmentation was highly correlated with ground-truth fluorescence (Extended Data Fig. 1c).

To reliably measure and calibrate physiological levels of intracellular lactate and glycolysis using laconic, we controlled intracellular lactate concentrations by permeabilizing human aortic endothelial cells (HAECs) and subjecting them to different concentrations of lactate. As shown in Extended Data Fig. 1 a, the $1 /$ FRET change is linear to 6 orders of magnitude of log lactate concentrations $\left(R^{2}=0.96\right)$. However, lactate export is a dynamic process in live cells that is regulated by the concentration gradient between the extra- and intra-cellular compartments ${ }^{13}$. Thus, in order to measure the glycolytic rate of the cell, lactate export was blocked through inhibition of monocarboxylate transporter 1 (MCT1), the major lactate transporter in endothelial cells ${ }^{14}$ (Fig. 1a). Upon MCT1 blockade by p-chloromercuribenzoic acid ( $p C M B A)$, we observed a continuous increase of lactate in individual cells, the slope of which we defined as the lactate production rate (LPR) (Fig. 1b), which reflects the overall glycolytic rate in a single endothelial cell. Furthermore, the single-cell LPR increased linearly, in agreement with bulk extracellular acidification measurements, as a function of extracellular glucose concentration (Fig. 1c). Employing a bootstrap algorithm simulating LPR, by drawing from ground truth fluorescence values and fitting slopes 
to the deep learning-measured fluorescence (Extended Data Fig. 1c), we estimated the absolute quantification error of LPR to be 5\% (see Methods, Extended Data Fig. 1d).

Consistently, the steady-state intracellular concentration of lactate measured by single-cell FRET was within the range estimated by bulk mass spectrometry ( 0-1.6 mM) (Fig. 1d). Corresponding to single-cell LPR, blockade of MCT1 with pCMBA ${ }^{13}$ also reduced extracellular acidification in a bulk bioenergetics flux assay (Extended Data Fig. 2a). Additionally, we were able to increase intracellular lactate at the single-cell level with glucose or oligomycin stimulation, and inhibit lactate production by 2-deoxyglucose, like those used in an extracellular flux glycolysis stress test at the bulk level (Extended Data Fig. 2b).

To test the LPR's ability to distinguish heterogeneous glycolytic rates in cell populations, which is critical for differentiating subpopulations, we mimicked hypoxic conditions in endothelial cells by treating with dimethyloxaloylglycine (DMOG). DMOG inhibits prolyl hydroxylase, thereby promoting the stabilization of transcription factor hypoxia inducible factor-1a (HIF-1a) ${ }^{2}$. We found that the single-cell LPR was elevated in DMOG-treated cells (Fig. 1e), in agreement with an increase in ECAR measured in bulk extracellular flux (Extended Data Fig. 2c). We next forced metabolic heterogeneity by mixing vehicle (DMSO)- or DMOG-treated cells that were previously labeled with either eBFP2 or mCherry fluorescent proteins, respectively (Fig. 1f). By assessing the LPR of each cell and then de-mixing by color (Fig. 1g), our method distinguished authentic metabolic subpopulations.

In addition to lactate, we further employed other genetically encoded FRET-based sensors to measure key glycolytic metabolites including pyruvate ${ }^{15}$, glucose ${ }^{16}$, and ATP ${ }^{17}$ to cross-validate these findings with DMOG. Pyruvate production, glucose uptake, and ATP levels were all increased in DMOGtreated cells relative to DMSO control (Fig. 1h-j). Notably, pyruvate and glucose have alternative fates and ATP is not exclusively produced by glycolysis, whereas lactate production is a definitive surrogate for glycolysis. The results not only validated our primary results using the laconic sensor, but also laid a solid foundation for the general application of our single-cell metabolism method across different metabolites.

\section{Measuring glycolytic rates of single endothelial cells in response to flow}

We then assessed single-cell glycolytic heterogeneity in a spatial context by exploiting the hemodynamic flow-dependent activation of endothelial cells, which are generally subject to different shear stresses depending on the local flow conditions in the vasculature ${ }^{18}$. Disturbed flow-induced cell activation leads to atherosclerosis at arterial branch points ${ }^{18}$, driven by low shear stress-induced endothelial glycolysis ${ }^{2,3}$, and abrogated by high shear stress which is known to repress glycolysis ${ }^{4}$ through reducing the expression of a key glycolytic enzyme, PFKFB3, in a transcription factor KLF2-dependent manner4. However, the ability to study flow-induced metabolic changes has so far been limited to extracellular flux without spatial resolution ${ }^{2}$. 
Using a cone and plate device to generate spatially heterogeneous shear forces over microscopic distances (Fig. 2a), we focused on an area at the flow boundary (indicated by endothelial elongation and alignment, Fig. 2b) to determine force-dependent metabolic rates. As shown in Fig. 2c, cells subjected to high shear stress had reduced LPR compared to those exposed to low shear stress, consistent with our previously published bulk data as measured by ECAR ${ }^{2}$. We next linked metabolism to a morphological phenotype (flow-induced endothelial cell elongation) at the single-cell level. By analyzing endothelial cells exposed to shear stress, we found a direct association between force-induced cell morphology and glycolytic rate. We plotted the LPR and shear stress as a function of cell length and found a negative correlation with LPR but a positive correlation with shear stress (Fig. 2d). At 20x magnification, cell length is strongly negatively correlated with LPR (Fig. 2e, f). This result was partially caused by the presence of external forces (shear stress), which could change metabolic rates independent of cell morphology, though shorter cells within the high shear stress region exhibit higher LPR (Fig. 2e).

\section{Glycolysis fuels the migration of single endothelial cells}

Endothelial cells migrate during angiogenesis or wound closure. This process requires energy, involves dramatic changes in cell shape, and is dependent on RhoA/ROCK-1 remodeling of the actin cytoskeleton ${ }^{5}$. In typical wound assays, single-cell migrations are heterogeneous where some cells are motile while others remain stationary ${ }^{19}$. Previously, it has been shown that endothelial cell migration requires glucose, whereas endothelial cell growth requires glutamine usage in oxidative phosphorylation ${ }^{1,20}$. However, it is not known whether individual migrating cells are more glycolytic than stationary cells, which could elucidate whether metabolism is a driver of heterogeneous cell migration in a disrupted cell monolayer. We first demonstrated that glucose is necessary for migration whereas mitochondrial metabolism is dispensable for wound closure (treatment with Antimycin A, Fig. 3a). The results indicated that cell migration is driven by glycolysis. Consistently, HAECs treated with DMOG, which increases glycolytic capacity in a HIF-1a-dependent manner ${ }^{21}$, enhanced migration and are able to close wounds faster than control (Fig. 3b).

However, it remains unknown whether individual cells that migrated further were more glycolytic, as activation of HIF-1a by DMOG could turn on thousands of genes ${ }^{2}$. In order to elucidate whether cell glycolysis fuels individual cell motility, we applied single-cell metabolic assays to analyze migrating endothelial cells in a wound assay. By tracking individual cell migrations (Fig. 3c) over the course of 12 hours, we confirmed that cells in the more stationary "tissue" migrated less than cells in the more migratory "wound", overall (Fig. 3d). We found that migratory cells, cells that traveled more than one cell length, were more glycolytic compared to stationary cells (Fig. 3e). We further found that cell migration distance is positively correlated with cell glycolysis (Fig. 3f), regardless of the final cell positions in the tissue or wound regions. These results suggest that the migration of endothelial cells is fueled by glycolysis, which is moreover proportional to work performed. 


\section{Singlecell intracellular lactate production reveals that RhoA activation increases endothelial glycolysis}

Having shown that glycolysis fuels cell migration, we next quantified the glycolytic rate during cell contraction, a key motile endothelial process that occurs over a short timespan (minutes) and is thus independent of transcriptional control. Because GTPase RhoA activation is central to induce the contractile apparatus of endothelial cells within minutes ${ }^{6}$, we hypothesized that controlled RhoA agonism would induce a glycolytic burst to power cell contractions. RhoA activation or inactivation in HAECs was achieved by overexpressing constitutively active RhoA (RhoA-Q63L) or dominant negative RhoA (RhoAT19N). As shown in Figure 4a, RhoA-Q63L (CA) increased single cell LPR compared to RhoA-T19N (DN). To remove possible batch effects, we next conjugated fluorescent protein eBFB2 to RhoA-T19N and fluorescent protein mScarlet-i to RhoA-Q63L, followed by cell mixing and single-cell LPR measurements. Deconvolution by color showed significantly increased glycolysis in RhoA-activated (mScarlet-i positive) HAECs when compared to RhoA-inactivated (eBFP2 positive) cells (Fig. 4b). Switching fluorescent labeling of RhoA-Q63L and RhoA-T19N had no effect on the results (Extended Data Fig. 3a). We next treated HAECs with lysophosphatidic acid (LPA), another well-known RhoA agonist ${ }^{22}$, which significantly increased single-cell LPR compared to untreated control (Fig. 4c). Furthermore, treatment with thrombin, a serine protease that rapidly stimulates RhoA and subsequently RhoA kinase- 1 (ROCK-1) ${ }^{23,24}$ to produce contractions and barrier breakdown, significantly increased LPR in 7 minutes, which was largely abrogated by the treatment of a specific ROCK-1 inhibitor, Y27632 (Fig. 4d). Consistently, glucose uptake and ATP levels were also elevated shortly after thrombin treatment (Fig. 4e). Thus, activation of GTPase RhoA causatively drives intracellular lactate production and glycolysis in single endothelial cells.

\section{RhoA promotes endothelial glycolysis to induce phosphorylation of cofilin and myosin light chain}

Because the activity of RhoA-depedent kinases requires ATP, we hypothesized that the downstream functions of RhoA/ROCK-1 are dependent on glycolysis. RhoA/ROCK-1 activation causes rapid phosphorylation of cofilin (CFL) which enacts reorganization of the actin cytoskeleton from cortical actin to stress fibers ${ }^{25,26}$. RhoA/ROCK-1 activity also causes phosphorylation of myosin light chain (MLC) which actively pulls on stress fibers, causing cell contractions and intercellular gaps in endothelial monolayers (failure of the endothelial paracellular barrier) as well as the reduction in monolayer impedance ${ }^{27-29}$. Phosphorylation of CFL and MLC occurs in 2-30 minutes with relaxation of the cell to its basal state after 1 hour as the stimulus is depleted ${ }^{26,30}$. To test our hypothesis, we used hexokinase inhibitor 2-deoxyglucose (2-DG) to block glycolysis. Indeed, 2DG significantly reduced basal CFL and MLC phosphorylation in HAECs and moreover, completely blocked thrombin-induced phosphorylation of CFL and MLC (Fig. 4f), demonstrating that RhoA/ROCK1 downstream effects are dependent on active glycolysis. Consistently, the degree of contraction, or change in monolayer impedance, was dependent on the concentration of glucose in the media, as measured by transendothelial electrical resistance (TEER) 
(Fig. 4g). Concordantly, depleting endothelial glycolysis with 2-DG also prevented thrombin-induced barrier failure by TEER (Fig. 4h). Furthermore, inhibition of ROCK-1 with Y27632 followed by thrombin treatment phenocopied pre-treatment with 2DG (Extended Data Fig. 3b). These data collectively demonstrated that RhoA/ROCK1-dependent glycolysis is required for the contractile machinery of endothelial cells.

\section{RhoA simulates endothelial contraction by triggering a glycolytic burst via glucose transporter SLC2A3/GLUT3}

We next determined the molecular mechanisms by which RhoA induces glycolysis to stimulate endothelial contraction. Since the increase in glycolysis occurs over a short timespan (measured at 7 minutes) following RhoA activation (Fig. 4d) and availability of glucose determines the degree of downstream phosphorylation and contraction (Fig. 4f, g), we hypothesized that glucose uptake plays a critical role in RhoA-induced glycolysis. Previous studies have shown that SLC2A1/GLUT1 is a major glucose transporter in endothelial cells. SLC2A1 contributes to the basal endothelial glycolytic capacity in the blood brain barrier ${ }^{31}$ and pulmonary vasculature ${ }^{32}$, as well as to increased glycolysis stimulated by chronic (hours) exposure to hypoxia and disturbed flow in large arteries ${ }^{2}$. We therefore first tested the role of SLC2A1. Unexpectedly, small interfering RNA (siRNA)-mediated SLC2A1 knockdown did not reduce thrombin-induced phosphorylation of CFL and MLC in HAECs (Extended Data Fig. 4a). At the same time, qRT-PCR analysis identified that SLC2A3 is the most abundant glucose transporter transcript in HAECs (Extended Data Fig. 4b). We therefore tested the role of SLC2A3 in RhoA-induced glycolysis. Our data showed that SLC2A3 knockdown by siRNAs reduced basal phosphorylation of CFL and MLC and significantly abrogated thrombin-induced phosphorylation of CFL and MLC with three separate siRNAs (Fig. 5a, Extended Data Fig. 4c,d).

Although SLC2A3 knockdown had no effect on basal endothelial glycolysis, it largely abolished the thrombin-induced glycolytic burst, as detected by single-cell LPR (Fig. 5b). In direct comparison, SLC2A1 knockdown led to a measurable decrease in LPR after thrombin stimulation but this decrease was far less than that of SLC2A3 knockdown (Extended Data Fig. 4e). Consistently, the thrombin-induced increase in glucose and ATP levels were abrogated by SLC2A3 knockdown (Extended Data Fig. 4f, g). Because the glycolytic burst could not be attributed to SLC2A3 transcriptional regulation given the acute response (measured within 7 minutes), we hypothesized that SLC2A3 is regulated post-transcriptionally by RhoA/ROCK-1. To test this hypothesis, we performed immunofluorescence imaging of SLC2A3 in thrombin-treated HAECs. Using total internal reflection fluorescence (TIRF) microscopy, which selectively illuminates membrane fluorophores close to the cover slip (within $100 \mathrm{~nm}$ ) ${ }^{33,34}$, we detected significantly increased intensity of SLC2A3 in granules near the plasma membrane of single HAECs following thrombin stimulation; such accumulation of SLC2A3 were abrogated by ROCK inhibitor Y27632, further suggesting the involvement of Rho/ROCK signaling pathway (Fig. 5c-d). In agreement with the SLC2A3-dependent glycolytic burst and phosphorylation of CFL and MLC, thrombin-induced endothelial 
contraction was significantly abrogated by SLC2A3 knockdown, demonstrated by increased monolayer impedance by TEER (Fig. 5e) and reduced intercellular gap size (gap size index, Fig. 5f), although the number of gaps was unchanged (gap index, Extended Data Fig. 4h) ${ }^{35}$. Furthermore, increasing the amount of extracellular glucose increased gap size and gap number, which was blunted by SLC2A3 knockdown (Extended Data Fig. 4i). Contraction (gap size index), but not gap number, was partially rescued under SLC2A3 knockdown by treatment with oligomycin to divert all glucose to be utilized via glycolysis (Extended Data Fig. 4j). Thrombin-induced phosphorylation of CFL and MLC were also significantly abrogated by SLC2A3 knockdown in human umbilical endothelial cells (HUVECs) (Extended Data Fig. 5a) and human lung microvascular cells (HMVECs) (Extended Data Fig. 5b). Thrombin-induced HUVEC contractions (as measured by cell size, Extended Data Fig. 5c) and HMVEC contractions (as measured by intercellular gaps, Extended Data Fig. 5d) were also significantly reduced by SLC2A3, indicating this pathway is conserved across multiple endothelial cell types.

The functional role of SLC2A3 in endothelial glycolysis and contraction is largely unknown. In order to explore the in vivo relevance of SLC2A3 in barrier regulation, we needed to first ascertain the expression profile of SIc2a1 and Slc2a3 in primary endothelial from different organs in mice. Gene Expression Omnibus datasets (GEOD) were downloaded from EndoDB, a database of curated endothelial transcriptomics datasets ${ }^{36}$. As shown in Extended Figure 6a, Slc2a3 has expression levels similar to Slc2a1 across multiple organs (normalized to Slc2a1). We next overexpressed SLC2A3 under the endothelial-specific CDH5/VE-cadherin promoter and evaluated vascular leak in mouse aortas. Mouse aortas, because to their intrinsic curvature, exhibit RhoA activation due to hemodynamic shear forces ${ }^{37}$. We employed a delivery system which integrates polyethylenimine (PEI) nanoparticles and plasmids with the endothelial-specific CDH5/VE-cadherin promoter. Intravenous injection of CDH5-driven, mScarlet-iexpressing plasmids resulted in mScarlet-i fluorescence in arterial endothelium, highlighted by colocalization with ve-cadh (which markes endothelium) adjacent to the lumen, but not the underlying media and adventitia (yellow signal in merge panel, Extended Fig. 6b), demonstrating endothelial-specific overexpression in mice. The same approach using CDH5-driven, SLC2A3-expresssing plasmids led to SLC2A3 overexpression in aortic endothelium but not in the media and adventitia in mice, measured by quantitative real-time PCR (Extended Data Fig. 6c). To demonstrate functional relevance of SLC2A3, mice were injected with PEI nanoparticles containing CDH5-SLC2A3 or CHD5-Ctrl plasmids 24 hours prior to sacrifice. 1 hour prior to sacrifice, Evans blue dye was injected retro-orbitally. Evans blue dye binds to albumin and transits the endothelial barrier in a paracellular fashion under conditions of diminished tight and adherent junctions and quantitatively measures the leakiness of mouse aortas ${ }^{38,39}$. Endothelial overexpression of SLC2A3 results in leakiness of aorta as quantified by Evans blue spectroscopy (OD 620), normalized to aorta weight after dye extraction (Extended Data Fig. 6d). This experiment suggests that SLC2A3 regulates the endothelial barrier in vivo. Collectively, these results demonstrated the importance of SLC2A3 on fueling RhoA/ROCK-1 downstream phosphorylation targets and its importance in both in vitro and in vivo barrier function. 


\section{Subcellular glycolysis is correlated with actin cytoskeletal remodeling}

After finding RhoA activation induces endothelial glycolysis and contraction within minutes at the singlecell scale (Fig. 4a-d), we sought to evaluate how glycolysis relates to morphological changes within individual endothelial cells with subcellular resolution. Reasoning that actin remodeling is dependent on high-energy phosphates, we hypothesized that localized changes in actin is correlated with glycolytic activity at the subcellular spatial scale. To test this hypothesis, we again stimulated actin remodeling with a RhoA agonist thrombin. By co-expressing laconic and Lifeact-RFP ${ }^{40}$ (an F-actin label) in endothelial cells, we measured LPR and actin remodeling in single endothelial cells with subcellular resolution at 40x magnification (Fig. 6a). Lifeact signal increases and shimmers at the edges of thrombin-induced contracting cells, indicating active actin remodeling. In order to visualize actin remodeling, we computed the maximum intensity projection of the difference in Lifeact signals in the 7 minutes following thrombin addition prior to performing the LPR assay (Fig. 6a, difference image). The LPR image was captured by performing pixel-wise calculation of the slope of the FRET signal after addition of pCMBA (Fig. 6a, LPR). As shown in the merged image, the LPR signal was spatially correlated with the Lifeact difference image (Fig. 6a, "LPR+difference" and "LPR+difference+nucleus"). The Lifeact difference and LPR images together demonstrated enhancement of signals on the contracting edge and center of the cell. To quantify the correlation, we calculated the degree of overlap using Manders' overlap coefficient (MOC) and Pearson's correlation coefficient (PCC). Thrombin-stimulated cells showed much higher levels of correlation between subcellular LPR and Lifeact than those of non-stimulated cells (Fig. 6b). This correlation was diminished by inhibition of ROCK (Y-27632), actin polymerization (Latrunculin B), and myosin II (para-amino-Blebbistatin) (Fig. 6b). Moreover, SLC2A3 knockdown reduced the correlation between Lifeact difference and LPR after thrombin treatment, indicating RhoA-dependent SLC2A3 activity is necessary for the co-occurrence of subcellular glycolysis and actin remodeling (Fig. 6c).

Similarly, we assessed subcellular glycolysis using Laconic in migrating endothelial cells. Here, we computed the Lifeact difference image in the 10 minutes prior to performing the LPR assay (Extended Data Fig. 7a, difference image). In migrating HAECs, we found that LPR and Lifeact difference were significantly correlated, compared to control (performed by randomizing the LPR images 10,000 times and computing the MOC or PCC) (Extended Data Fig. 7b). These results indicate spatial cytoskeletal remodeling and glycolysis are correlated in multiple types of endothelial motilities.

To determine whether subcellular LPR was spatially related to RhoA, we next evaluated RhoA activity using a FRET-based biosensor RhoA-Flare ${ }^{41}$ in single contractile endothelial cells. We found enhanced RhoA activity and increased LPR co-located in similar areas following thrombin stimulation: the center of the cells (Fig $6 \mathrm{~d}$ ) as well as along the edges of the contractile aspects of the cell (Fig. $6 \mathrm{~d}$-f, and Extended Data Fig. 7c-I). In contrast, where the cell edge did not move, there was no FRET (RhoA activity) (Extended Data Fig. 7i). Edges with persistent contraction consistently demonstrated high RhoA-Flare FRET (Extended Data Fig. 7j). The similar distribution of RhoA activity and LPR was further confirmed by comparing stationary edges vs contractile edges across multiple cells, averaged together (Fig. $6 \mathrm{~g}$ ). This data revealed that the non-uniform localized distribution of metabolism correlates with cytoskeleton 
remodeling at the subcellular level. These results further showed co-localization between LPR and RhoA activities, suggesting that an endothelial cell generates energy using glycolysis to drive actin remodeling through GTPase RhoA.

Altogether, our single-cell metabolic assays revealed molecular mechanisms that RhoA/ROCK-1 activates SLC2A3 translocation to trigger a glycolytic burst to power the endothelial contractile apparatus across multiple endothelial types (Fig. 6h). Furthermore, subcellular analysis of LPR revealed the non-uniform, localized distribution of glycolysis at site of actin turnover and RhoA activity, indicating RhoA signaling coordinates energy production through glycolysis with energy consumption through cytoskeletal remodeling to control cell motility.

\section{Discussion}

Most studies measure cell metabolism using bulk assays. However, individual cells are inherently heterogeneous. Metabolic heterogeneity in endothelial cells dictates vascular activation and growth ${ }^{42,43}$, during T-cell differentiation controls cell function in infection ${ }^{44,45}$, within tumors affects responses to anticancer treatments ${ }^{46}$, and among yeast cells results in distinct subpopulations in response to sudden changes of substrate availability ${ }^{47}$, to name a few. Reprogrammed metabolic rates to support different cellular functions has rightfully gained attention but experimental methods to directly evaluate heterogeneous populations remain in infancy. To decipher the metabolic states of individual cells, we used a FRET-based metabolic imaging method to measure single-cell metabolism with subcellular resolution. Our single-cell metabolism method is complementary to existing ensemble metabolic assays and broadly applicable to different cell types. With this method, we were able to investigate the underlying molecular mechanisms of glycolysis in endothelial cells. This method enabled us to link heterogeneous glycolytic states to GTPase RhoA-activated phenotypes across variable time scales (low shear stress: days, migration: hours, and contraction: minutes) in individual endothelial cells. This method also allowed us to explore spatially heterogeneous metabolism and motility within individual cells. Given the feasibility, reproducibility, and robustness of this approach, it should have widespread applications to study metabolic support of single-cell functions in cell biology.

In addition to identifying single-cell metabolic heterogeneity, we found non-uniform distributions of glycolytic rates within single motile cells, specifically at sites of actin turnover and RhoA activity. RhoAcoupled glycolysis and contractile machinery demonstrated here also support an emerging paradigm that cellular metabolism and cytoskeleton architecture are intimately coordinated in an "energetic metabolon". Our results also suggest that an endothelial cell is energetically frugal and samples the environment for energetic precursors only when necessary: here, the cell employs RhoA/ROCK- 1 to couple energy production with acute energy-consuming responses such as cytoskeletal remodeling to enable contraction. This could be functionally essential, as the continued presence of high energy phosphates in a cell may make controlling enzymatic phosphorylation difficult. Thus, having a local, segregated pool of transmembrane receptors may enable digital-like spatial control of phosphorylation ${ }^{48}$. Co-localization of 
glycolytic enzymes with energetic demand could provide an efficient way for the cell to not only temporally determine when energy is produced, but spatially control where energy is consumed, preventing futile reactions, avoiding energy over-production, and overcoming diffusion limitation. "Glycolytic metabolons" have been suggested in endothelial cells, with increased ATP in in the perinuclear cytosol and lamellipodial ruffles ${ }^{42,49}$, and evidence that glycolytic enzymes bind to the actin cytoskeleton ${ }^{50}$. Here, using single-cell metabolic assays, we directly visualized the highly localized, enriched lactate production in subcellular endothelial edges and perinuclear sites, providing evidence to functionally support the "glycolytic metabolon" theory. Further studies linking the subcellular glycolytic rate and distribution of glycolytic machinery are needed to strengthen and validate this theory.

Integrating our single-cell metabolic method with classic molecular and functional analyses also allowed us to discover the causal role of RhoA in stimulating a SLC2A3-dependent glycolytic burst necessary for endothelial contraction. Although it is commonly recognized that SLC2A1/GLUT1 is a major glucose transporter in endothelial cells across diverse vascular beds and contributes to basal glycolysis under chronic transcriptional activation 2,31,32,51, our results revealed the critical role of SLC2A3/GLUT3 in triggering a metabolic burst, defined by a quick increase in glucose uptake, glycolysis, and ATP level. ATP budget and nutrient availability plays an important role in cellular decision making ${ }^{52-54}$, including certain endothelial behaviors ${ }^{20,43,54,55}$. Using this mechanism, a single endothelial cell can augment its glycolytic potential even further with SLC2A3 transmembrane recruitment during acute stimuli in cellular processes requiring an immediate energy burst. We also demonstrated that vascular leak was increased in an endothelial overexpression model of SLC2A3 in vivo. In highly-glycolytic platelets where SLC2A3 is enriched, thrombin (a potent platelet activator) can stimulate SLC2A3 plasma membrane translocation ${ }^{56}$, suggesting a conserved molecular function. Exactly how RhoA/ROCK-1 causes transmembrane expression of SLC2A3 requires further exploration. Investigation of SLC2A3's role in regulating vascular pathophysiology could bolster the case that modulating signal transduction by interfering with cellular energetics could be a fruitful path to novel pharmacotherapeutics in vascular pathologies. The physiological role of endothelial SLC2A3 in vascular homeostasis and disease in vivo deserves future investigation.

Importantly, we found lactate production to be highest at the cell edge and center of contractile cells. Though we attribute this to energy demand at locations of high RhoA activity, one could hypothesize that lactate could also be produced locally for usage for other cellular processes such as histone lactylation within the nucleus ${ }^{57}$. Perhaps the increase of local glycolysis could act as a signal for the cell to adapt to a changing environment, thus imprinting acute stimuli into cellular memory. Future investigation of subcellular endothelial glycolysis would benefit from tracing high energy phosphates from where they are produced, to sites where they are consumed, including consideration of nuclear lactate as it corresponds to gene regulation. For instance, FRET biosensors can be further engineered with a nuclear localization sequence (NLS) to facilitate nuclear targeting while membrane targeting of theses sensors might be achieved by adding the palmitoylation tag from $\mathrm{GAP} 43^{58}$ or the myristoylation tag from $\mathrm{Src}^{59}$. 
In summary, we investigated spatially heterogeneous endothelial motilities by developing a single-cell imaging assay to measure glycolysis with subcellular resolution. We demonstrated that endothelial migration and contraction, critical endothelial phenotypes, were driven by glycolysis at the single-cell and subcellular levels. We found energy-demanding cytoskeletal remodeling to be conducted by RhoA through SLC2A3-mediated glucose uptake and glycolysis. Altogether, our single-cell metabolic assays and mechanistic findings demonstrated the critical interplay between signaling, metabolism, and motility in dictating non-uniform phenotypes of individual endothelial cells.

\section{Declarations}

\section{Acknowledgements}

This work was supported by NIH grants R00Al106941 (JH), R21Al120010 (JH), R01HL138223 (YF), and R01HL136765 (YF), NIH New Innovator Award DP2Al144245 (JH), T32EB009412 (DLH), T32HL007381 (DLH), F32HL134288 (DW), NIH Pathway to Independence Award K99HL145113 (DW), NVIDIA GPU Grant (DW), CSCTR Early Career Development Award (DW), and NSF Career Award 1653782 (JH).

\section{Author contributions}

D.W. and D.L.H. planned and executed experiments, analyzed data and interpreted results. T.S. analyzed data. C.Y., A.M., T.S., R.H., and Z.Z. performed experiments. J.H. and Y.F. planned experiments and interpreted results. G.M. helped design experiments. D.W., D.L.H., J.H., and Y.H. wrote and edited the manuscript.

\section{Declaration of interests}

There authors declare no competing interests.

\section{Data and Code Availability}

The code generated during this study is available at https://github.com/wulab-code/laconic without restriction. Supporting data of the findings of this study are available upon reasonable request from the corresponding author.

\section{References}

1. Kim, B., Li, J., Jang, C. \& Arany, Z. Glutamine fuels proliferation but not migration of endothelial cells. $E M B O J$ 36, 2321-2333 (2017). 
2. Wu, D., et al. HIF-1 alpha is required for disturbed flow-induced metabolic reprogramming in human and porcine vascular endothelium. Elife 6(2017).

3. Feng, S., et al. Mechanical Activation of Hypoxia-Inducible Factor 1alpha Drives Endothelial Dysfunction at Atheroprone Sites. Arterioscler Thromb Vasc Bio/37, 2087-2101 (2017).

4. Doddaballapur, A., et al. Laminar shear stress inhibits endothelial cell metabolism via KLF2-mediated repression of PFKFB3. Arterioscler Thromb Vasc Biol 35, 137-145 (2015).

5. van Nieuw Amerongen, G.P., Koolwijk, P., Versteilen, A. \& van Hinsbergh, V.W. Involvement of RhoA/Rho kinase signaling in VEGF-induced endothelial cell migration and angiogenesis in vitro. Arterioscler Thromb Vasc Biol 23, 211-217 (2003).

6. Spindler, V., Schlegel, N. \& Waschke, J. Role of GTPases in control of microvascular permeability. Cardiovasc Res 87, 243-253 (2010).

7. Falk, T., et al. U-Net: deep learning for cell counting, detection, and morphometry. Nat Methods 16, 6770 (2019).

8. Moen, E., et al. Deep learning for cellular image analysis. Nat Methods 16, 1233-1246 (2019).

9. Van Valen, D.A., et al. Deep Learning Automates the Quantitative Analysis of Individual Cells in LiveCell Imaging Experiments. PLoS Comput Bio/ 12, e1005177 (2016).

10. Ronneberger, O., Fischer, P. \& Brox, T. U-Net: Convolutional Networks for Biomedical Image Segmentation. 234-241 (Springer International Publishing, Cham, 2015).

11. San Martin, A., et al. A genetically encoded FRET lactate sensor and its use to detect the Warburg effect in single cancer cells. PLoS One 8, e57712 (2013).

12. Csurka, G.L., Diane. What is a good evaluation measure for semantic segmentation? IEEE Trans. Pattern Anal. Mach. Intell. 26(2013/01/01).

13. Halestrap, A.P. Monocarboxylic acid transport. Compr Physio/3, 1611-1643 (2013).

14. Sonveaux, P., et al. Targeting the lactate transporter MCT1 in endothelial cells inhibits lactate-induced HIF-1 activation and tumor angiogenesis. PLoS One 7, e33418 (2012).

15. San Martin, A., et al. Imaging mitochondrial flux in single cells with a FRET sensor for pyruvate. PLoS One 9 , e85780 (2014).

16. Takanaga, H., Chaudhuri, B. \& Frommer, W.B. GLUT1 and GLUT9 as major contributors to glucose influx in HepG2 cells identified by a high sensitivity intramolecular FRET glucose sensor. Biochim Biophys Acta 1778, 1091-1099 (2008).

17. Imamura, H., et al. Visualization of ATP levels inside single living cells with fluorescence resonance energy transfer-based genetically encoded indicators. Proc Natl Acad Sci U S A 106, 15651-15656 (2009).

18. Davies, P.F. Flow-mediated endothelial mechanotransduction. Physiol Rev 75, 519-560 (1995).

19. Mayor, R. \& Etienne-Manneville, S. The front and rear of collective cell migration. Nat Rev Mol Cell Biol 17, 97-109 (2016). 
20. Diebold, L.P., et al. Mitochondrial complex III is necessary for endothelial cell proliferation during angiogenesis. Nature Metabolism 1, 158-171 (2019).

21. Mole, D.R., et al. 2-Oxoglutarate analogue inhibitors of hif prolyl hydroxylase. Bioorganic \& Medicinal Chemistry Letters 13, 2677-2680 (2003).

22. van Nieuw Amerongen, G.P., Vermeer, M.A. \& van Hinsbergh, V.W. Role of RhoA and Rho kinase in lysophosphatidic acid-induced endothelial barrier dysfunction. Arterioscler Thromb Vasc Bio/20, E127-133 (2000).

23. van den Biggelaar, M., et al. Quantitative phosphoproteomics unveils temporal dynamics of thrombin signaling in human endothelial cells. Blood 123, e22-36 (2014).

24. Vouret-Craviari, V., Grall, D. \& Van Obberghen-Schilling, E. Modulation of Rho GTPase activity in endothelial cells by selective proteinase-activated receptor (PAR) agonists. J Thromb Haemost $\mathbf{1}$, 1103-1111 (2003).

25. Blanchoin, L., Boujemaa-Paterski, R., Sykes, C. \& Plastino, J. Actin dynamics, architecture, and mechanics in cell motility. Physiol Rev 94, 235-263 (2014).

26. Fazal, F., et al. Essential role of cofilin-1 in regulating thrombin-induced RelA/p65 nuclear translocation and intercellular adhesion molecule 1 (ICAM-1) expression in endothelial cells. J Biol Chem 284, 21047-21056 (2009).

27. Goddard, L.M. \& Iruela-Arispe, M.L. Cellular and molecular regulation of vascular permeability. Thromb Haemost 109, 407-415 (2013).

28. Mehta, D. \& Malik, A.B. Signaling mechanisms regulating endothelial permeability. Physiol Rev 86, 279-367 (2006).

29. Vandenbroucke, E., Mehta, D., Minshall, R. \& Malik, A.B. Regulation of endothelial junctional permeability. Ann N Y Acad Sci 1123, 134-145 (2008).

30. van Nieuw Amerongen, G.P., van Delft, S., Vermeer, M.A., Collard, J.G. \& van Hinsbergh, V.W. Activation of RhoA by thrombin in endothelial hyperpermeability: role of Rho kinase and protein tyrosine kinases. Circ Res 87, 335-340 (2000).

31. Tang, M., et al. Brain microvasculature defects and Glut1 deficiency syndrome averted by early repletion of the glucose transporter-1 protein. Nat Commun 8, 14152 (2017).

32. Fessel, J.P., et al. Metabolomic analysis of bone morphogenetic protein receptor type 2 mutations in human pulmonary endothelium reveals widespread metabolic reprogramming. Pulm Circ 2, 201-213 (2012).

33. Sasmal, D.K., et al. TCR-pMHC bond conformation controls TCR ligand discrimination. Cell Mol Immunol 17, 203-217 (2020).

34. Sasmal, D.K., Pulido, L.E., Kasal, S. \& Huang, J. Single-molecule fluorescence resonance energy transfer in molecular biology. Nanoscale 8, 19928-19944 (2016).

35. Fraccaroli, A., et al. Endothelial alpha-parvin controls integrity of developing vasculature and is required for maintenance of cell-cell junctions. Circ Res 117, 29-40 (2015). 
36. Khan, S., et al. EndoDB: a database of endothelial cell transcriptomics data. Nucleic Acids Res 47, D736-D744 (2019).

37. Wu, C., et al. Mechanosensitive PPAP2B Regulates Endothelial Responses to Atherorelevant Hemodynamic Forces. Circ Res 117, e41-53 (2015).

38. Yao, L., Xue, X., Yu, P., Ni, Y. \& Chen, F. Evans Blue Dye: A Revisit of Its Applications in Biomedicine. Contrast Media Mol Imaging 2018, 7628037 (2018).

39. Yang, Q., et al. PRKAA1/AMPKalpha1-driven glycolysis in endothelial cells exposed to disturbed flow protects against atherosclerosis. Nat Commun 9, 4667 (2018).

40. Riedl, J., et al. Lifeact: a versatile marker to visualize F-actin. Nat Methods 5, 605-607 (2008).

41. Pertz, O., Hodgson, L., Klemke, R.L. \& Hahn, K.M. Spatiotemporal dynamics of RhoA activity in migrating cells. Nature 440, 1069-1072 (2006).

42. De Bock, K., et al. Role of PFKFB3-driven glycolysis in vessel sprouting. Cell 154, 651-663 (2013).

43. Wilhelm, K., et al. FOXO1 couples metabolic activity and growth state in the vascular endothelium. Nature 529, 216-220 (2016).

44. Karmaus, P.W.F., et al. Metabolic heterogeneity underlies reciprocal fates of TH17 cell stemness and plasticity. Nature 565, 101-105 (2019).

45. Bantug, G.R., Galluzzi, L., Kroemer, G. \& Hess, C. The spectrum of T cell metabolism in health and disease. Nat Rev Immunol 18, 19-34 (2018).

46. Hensley, Christopher T., et al. Metabolic Heterogeneity in Human Lung Tumors. Cell 164, 681-694 (2016).

47. van Heerden, J.H., et al. Lost in transition: start-up of glycolysis yields subpopulations of nongrowing cells. Science 343, 1245114 (2014).

48. Huang, J., et al. A single peptide-major histocompatibility complex ligand triggers digital cytokine secretion in CD4(+) T cells. Immunity 39, 846-857 (2013).

49. Eelen, G., et al. Endothelial Cell Metabolism. Physiol Rev 98, 3-58 (2018).

50. Masters, C. Interactions between glycolytic enzymes and components of the cytomatrix. J Cell Biol 99, 222s-225s (1984).

51. Huang, Y., et al. Normal glucose uptake in the brain and heart requires an endothelial cell-specific HIF1alpha-dependent function. Proc Natl Acad Sci U S A 109, 17478-17483 (2012).

52. Herzig, S. \& Shaw, R.J. AMPK: guardian of metabolism and mitochondrial homeostasis. Nat Rev Mol Cell Biol 19, 121-135 (2018).

53. Saxton, R.A. \& Sabatini, D.M. mTOR Signaling in Growth, Metabolism, and Disease. Cel/ 168, 960-976 (2017).

54. Kuo, A., Lee, M.Y. \& Sessa, W.C. Lipid Droplet Biogenesis and Function in the Endothelium. Circ Res 120, 1289-1297 (2017).

55. Yu, P., et al. FGF-dependent metabolic control of vascular development. Nature 545, 224-228 (2017). 
56. Sorbara, L.R., et al. Thrombin-induced translocation of GLUT3 glucose transporters in human platelets. Biochem J 328 ( Pt 2), 511-516 (1997).

57. Zhang, D., et al. Metabolic regulation of gene expression by histone lactylation. Nature $574,575-580$ (2019).

58. Matsuda, T. \& Cepko, C.L. Controlled expression of transgenes introduced by in vivo electroporation. Proc Natl Acad Sci U S A 104, 1027-1032 (2007).

59. Rhee, J.M., et al. In vivo imaging and differential localization of lipid-modified GFP-variant fusions in embryonic stem cells and mice. Genesis 44, 202-218 (2006).

\section{Figures}



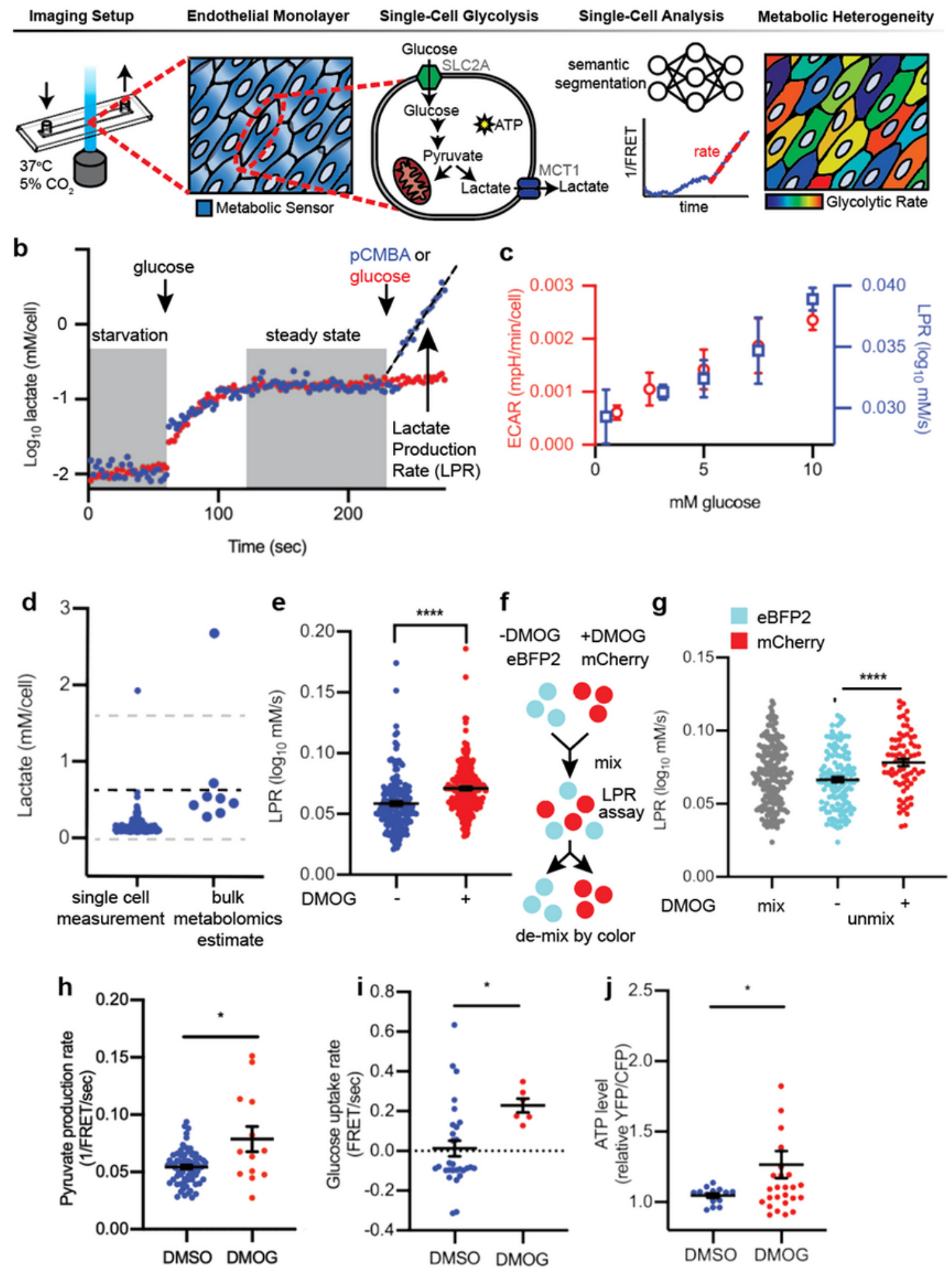

\section{Figure 1}

Single-cell lactate production rate (LPR) distinguishes metabolically heterogeneous subpopulations. a, Schematic of single-cell metabolism quantification by deep learning. Endothelial cells were plated into imaging chambers capable of fluid exchange with controlled temperature and $\mathrm{CO} 2$. Once endothelial cells grew to form a monolayer, metabolite sensors were genetically introduced to measure glycolysis. Within each cell, glucose is converted to pyruvate and consumed by mitochondria or fermented into lactate and 
exported via MCT1, thus generating ATP. Deep learning-enabled semantic segmentation was used for linking cells into a 1/FRET time series for quantification of intracellular lactate over time to reveal metabolic heterogeneity. b, Following starvation, glucose was injected followed by glucose and MCT1 inhibitor pCMBA (blue dots) or glucose alone (red dots). The slope (black fitted line) of each cell is the lactate production rate (LPR), filtered for fitting with $R 2$ values $\geq 0.9$. c, Single-cell LPR and per cell ECAR vs extracellular glucose concentration $(n=7,226,43,200,206$ cells for LPR left to right, $n=4,3,4,3,3$ wells for ECAR left to right; error bars are SEM). d, Comparison of steady-state lactate levels $(n=167$ cells) with metabolomics $(n=8)$. The dotted black line is the mean metabolomics $(n=8)$ estimate of intracellular lactate measured by mass spectrometry. Gray dotted lines are standard error of the mean (SEM). e, LPR of vehicle DMSO ("-") or DMOG ("+") treated endothelial cells ( $n=164$ "-", 256 " +"; error bars were SEM). $f$, eBFP2 or mCherry cells were treated with DMSO or DMOG, then mixed and imaged together; $g$, the LPR is then deconvoluted by color in ( $n=166$ eBFP2, $n=79$ mCherry; error bars are SEM). $h-j$, Pyruvate production rate ( $h, n=67,13)$, glucose uptake rate $(i, n=29,6)$, and ATP levels $(j, n=14,29)$ in HAECs treated overnight with DMSO or DMOG measured using genetically encoded sensors. ${ }^{*} p<0.05$,

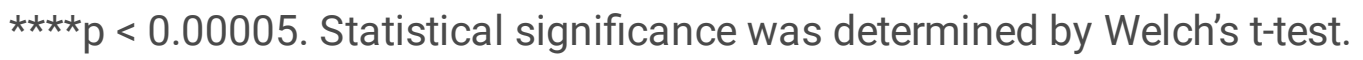
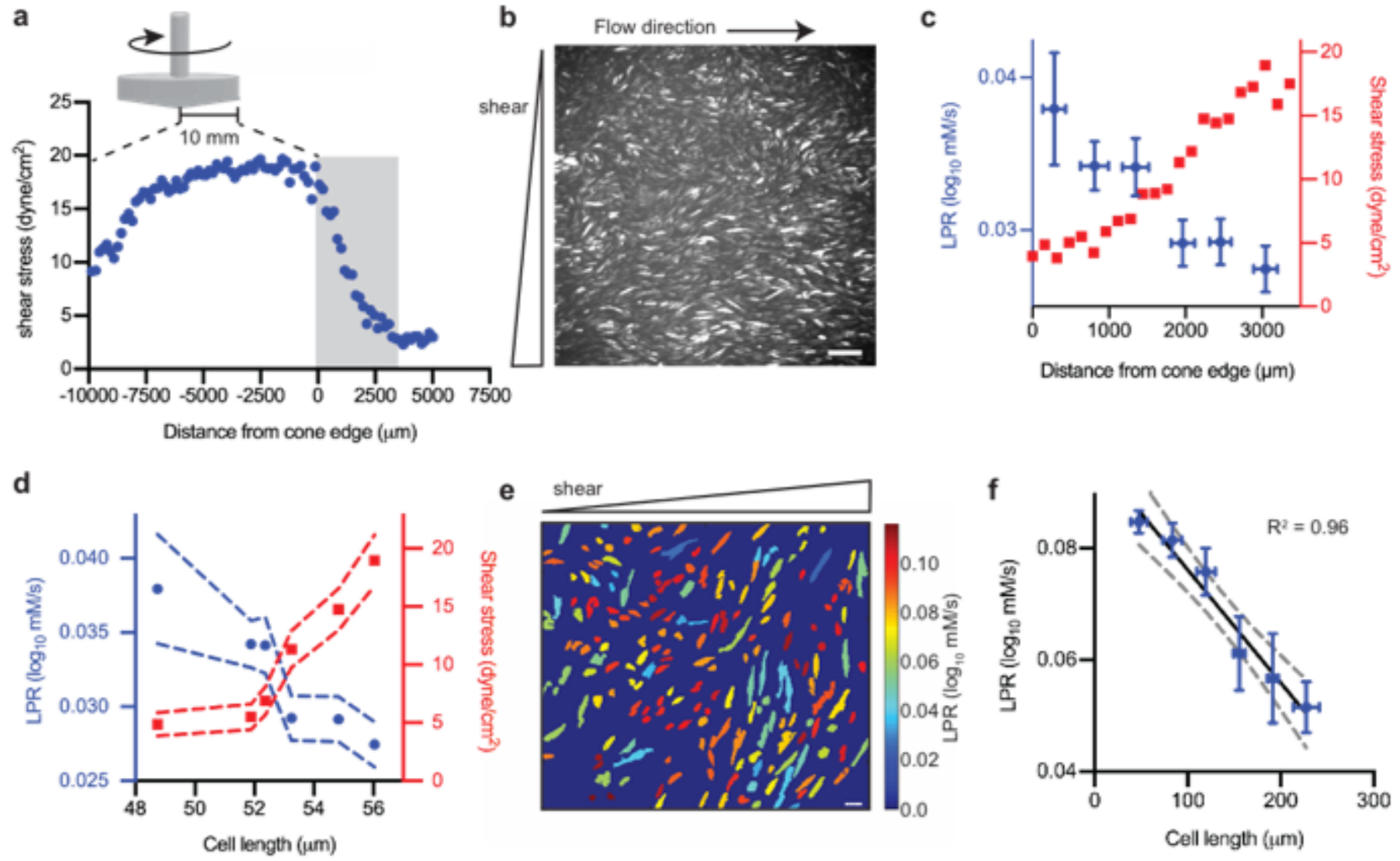

\section{Figure 2}

Endothelial LPR in response to shear stress is heterogeneous. a, A cone and plate device was used to generate shear stress as measured by particle image velocimetry, with gray bar indicating area of interrogation. b, The corresponding laconic fluorescence image at the gray zone in a. Scale bar $=165 \square \mathrm{m}$. c, LPR and shear stress vs distance from cone edge $(n=27,47,34,34,35,33$ left to right; error bars are 
SEM). d, Replot of c, with LPR and shear stress as a function of cell length. e, The LPR of segmented endothelial cells subjected to flow (color from cold to hot indicated the increase of LPR in each cell). Scale bar $=44 \square \mathrm{m} . \mathrm{f}$, Major axis cell length of endothelial cells (e) plotted against measured LPR (cell number $n=103,47,18,8,8,4$ low to high cell length; error bars are SEM). Standard deviation of regression coefficients for $\mathrm{R} 2$ for $(\mathrm{f})$ is 0.074 .
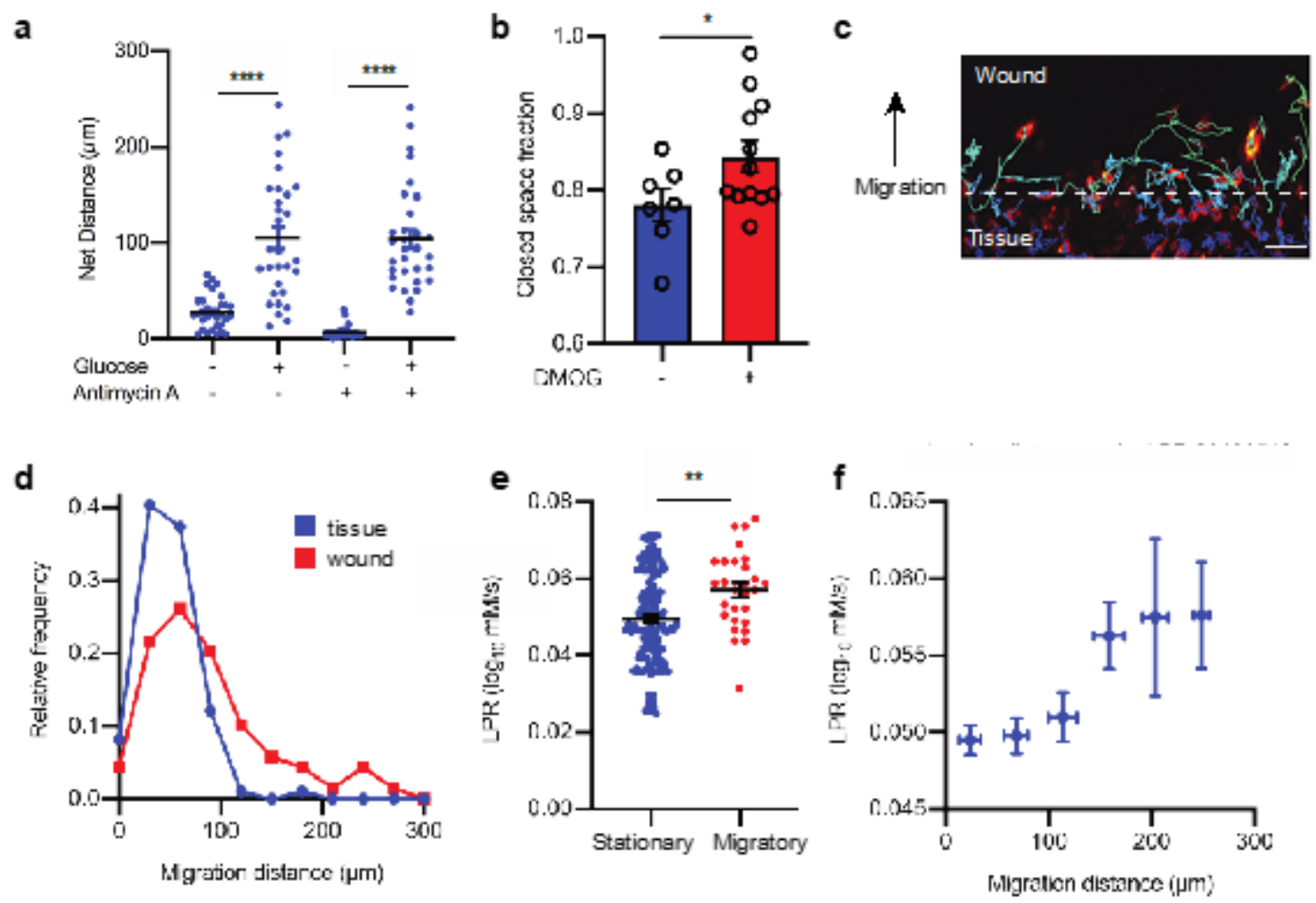

Figure 3

Single-cell metabolism links migratory cell phenotypes to glycolysis states. a, Migration distance of HAECs with or without glucose and with or without antimycin A $(n=31$ cells each condition; error bars are SEM). b, Migration scratch assay quantification with and without DMOG treatment ( $n=7$ and 12 , respectively; error bars are SEM). c, Migrating endothelial cells (false color) were tracked for 48 hours following wound formation. Scale bar $=150 \mathrm{am}$. d, Frequency distribution of cell migration distance in the wound vs tissue. e, The LPR of cells that migrated less than $50 \mathrm{am}$ compared to cells that migrated more than $200 \square \mathrm{m}$ (cell number $\mathrm{n}=141$ and 28, respectively; error bars are SEM). $\mathrm{f}$, The LPR change as a function of cell migrated distance $(n=114,118,42,12,8$ from low to high migration distance; error bars are SEM). ${ }^{\star} p<0.05,{ }^{*} p<0.005,{ }^{\star * \star *} p<0.00005$. Statistical significance was determined by Welch's ttest. 


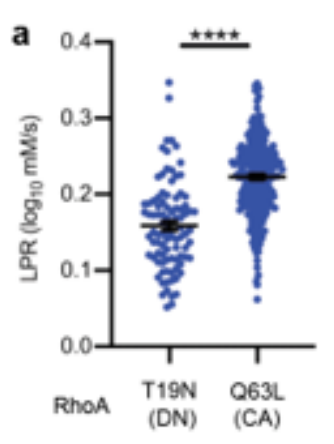

e

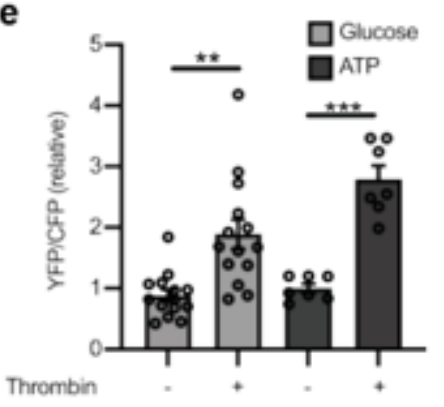

g

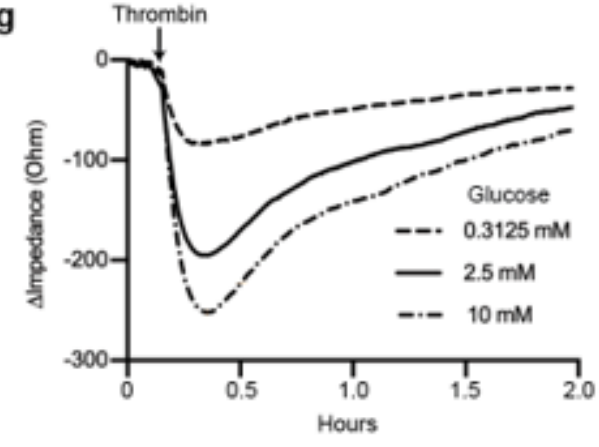

f

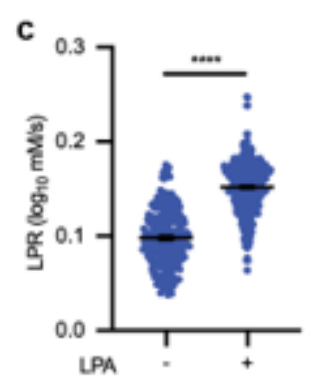

d
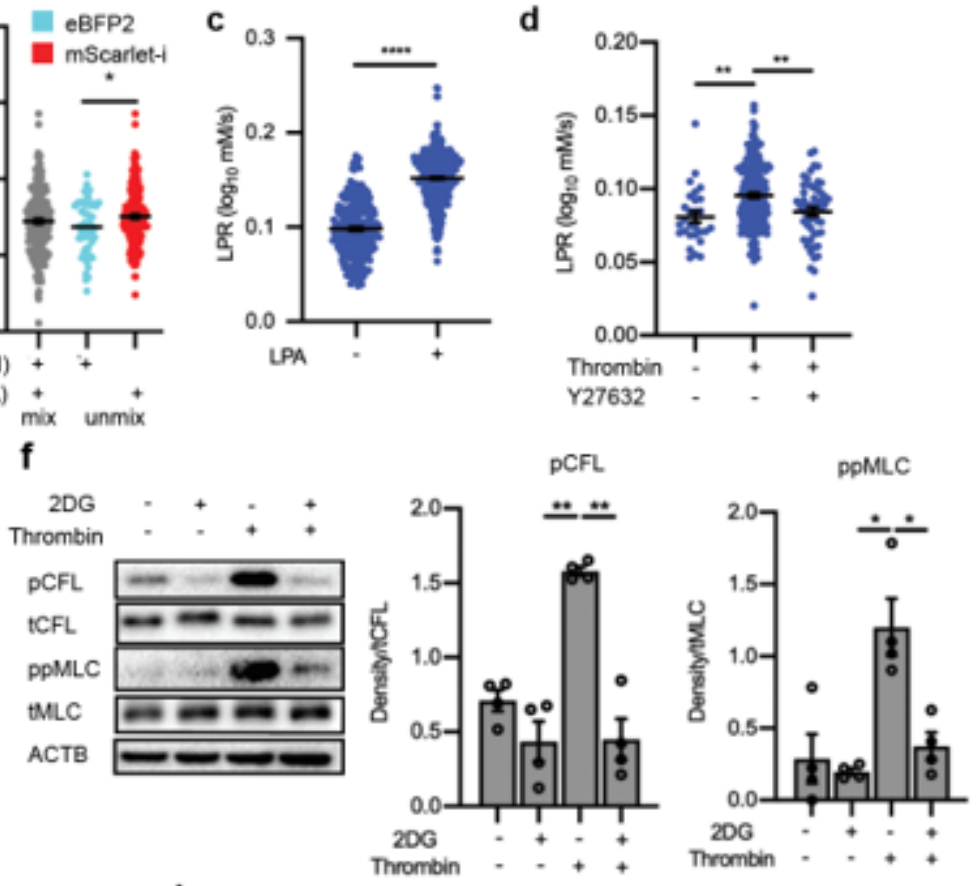

h

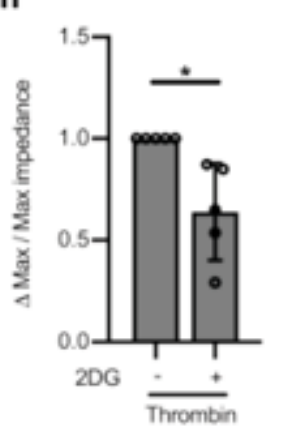

Figure 4

Single-cell imaging of lactate production reveals RhoA drives endothelial cell glycolysis, which is necessary for contractions. a, LPR of HAECs transfected with either RhoA-T19N (dominant negative "DN" form) or RhoA-Q63L (constitutively active "CA" form). ( $n=91,250$ respectively). b, RhoA-Q63L-mScarlet-i transfected ECs have increased LPR compared to those transfected with RhoA-T19N-eBFP after unmixing by color ( $\mathrm{n}=52$ and 174, respectively). c, LPR of HAECs treated with control (DMSO "-") or 10 [m of lysophosphatidic acid for $30 \mathrm{~min}$. ( $n=244$ and 423 respectively). $d$, Thrombin induced LPR $(n=171)$ compared to control $(n=28)$ which is suppressed with Y27632 $(n=51)$. e, ATP and glucose levels before and after treatment with thrombin measured by FRET ( $n=7$ cells ATP, $n=14$ cells glucose). f, Western blot of 2DG pre-treatment in the presence or absence of thrombin and phosphorylation of cofilin and MLC $(n=4) . g$, TEER trace of HAECs after thrombin treatment in different extracellular glucose conditions. $h$, Inhibition of hexokinase with 2-DG reduces thrombin-induced permeability as measured by TEER $(n=5$ each)., ${ }^{\star} p<0.05,{ }^{\star \star} p<0.005,{ }^{* \star \star} p<0.00005$. Statistical significance determined by Welch's t-test $(a, b, c$, $\mathrm{e}, \mathrm{h}$ ) or by one-way ANOVA followed by Bonferroni test $(\mathrm{d}, \mathrm{f})$. 

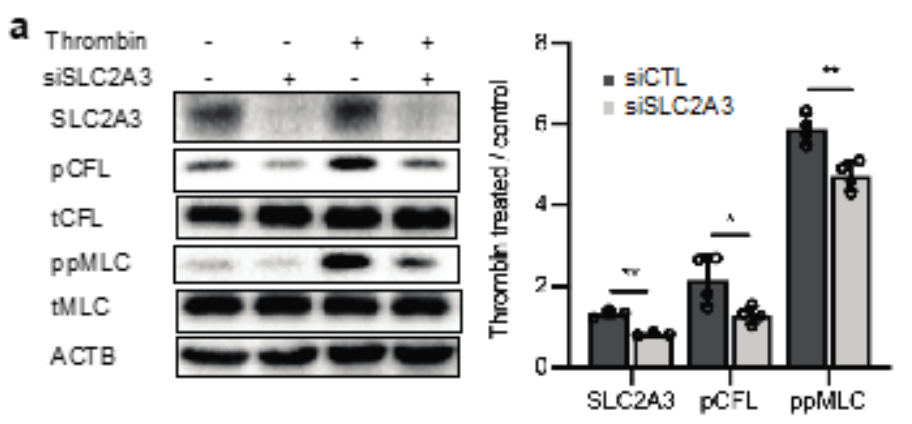

C
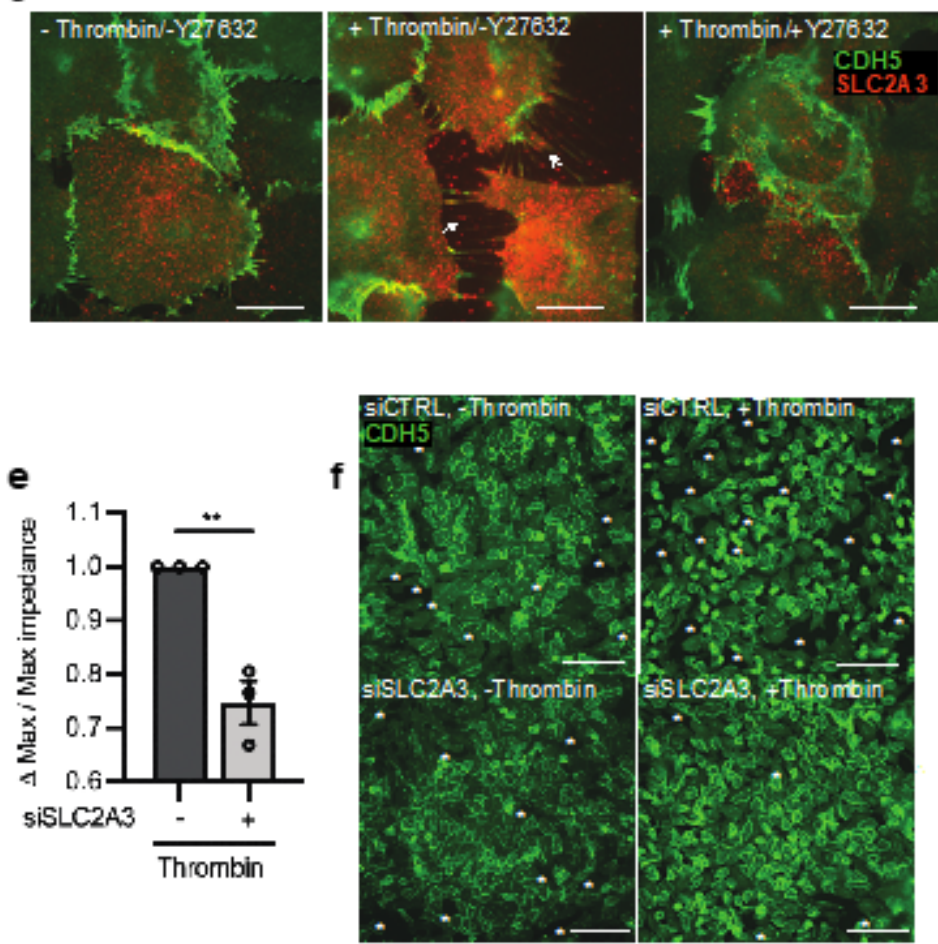

b

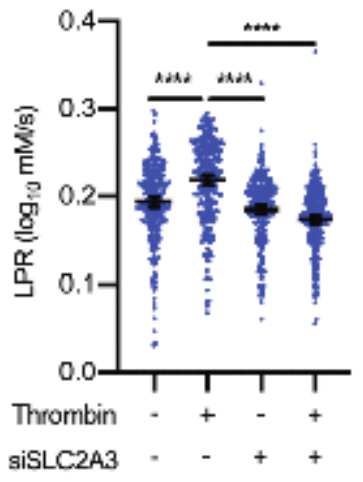

d
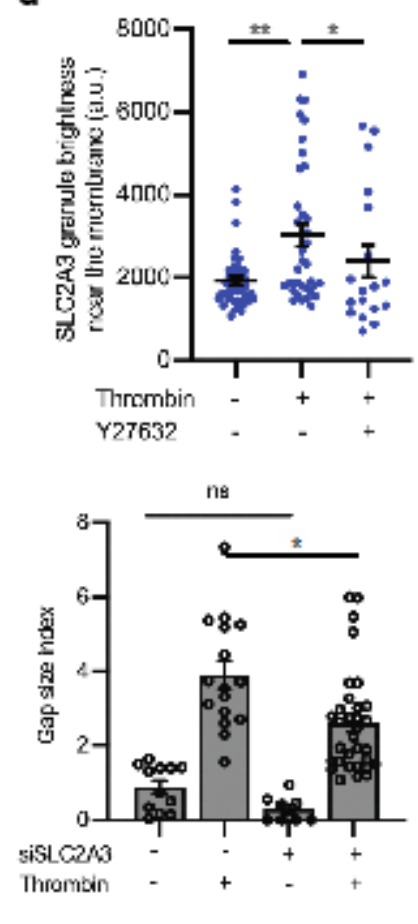

\section{Figure 5}

RhoA induces glucose transporter SLC2A3 translocation which drives subcellular glycolysis in thrombin stimulated contractile endothelial cells. a, Western blot of phospho-cofilin and phospho-MLC in the presence or absence of siRNA targeted towards SLC2A3 and thrombin treatment $(n=4$ all). b, LPR of siRNA targeted towards SLC2A3 or control with and without thrombin treatment $(n=277,263,275,278$ from left to right). c, Representative immunofluorescence of membrane markers CDH5 and SLC2A3 in thrombin treated cells causes SLC2A3 granule localization to membrane spikes, which is inhibited by Y27632, by TIRF microscopy. Scale bar $=5 \square \mathrm{m}$. d, Quantification of SLC2A3 granule brightness near the membrane of (c) (a.u. = arbitrary units, $n=36,36,17$ images from left to right). e, TEER measurement of thrombin-induced permeability changes in the presence or absence of siRNA targeted towards SLC2A3 ( $n$ = 4). $\mathrm{f}$, Representative immunofluorescence of $\mathrm{CDH} 5$ and intercellular gaps, as quantified by gap size index, in HAECs treated with control or siRNA targeted towards SLC2A3 followed by thrombin treatment ( $\mathrm{n}$ $=12,16,8,32$ images from left to right). Scale bar $=150 \square \mathrm{m} .{ }^{* \star \star \star} \mathrm{p}<0.00005,{ }^{*} \mathrm{p}<0.005,{ }^{\star} \mathrm{p}<0.05$. 
Statistical significance determined by Welch's t-test $(a, e, f)$ or by one-way ANOVA followed by Bonferroni test (b, d).
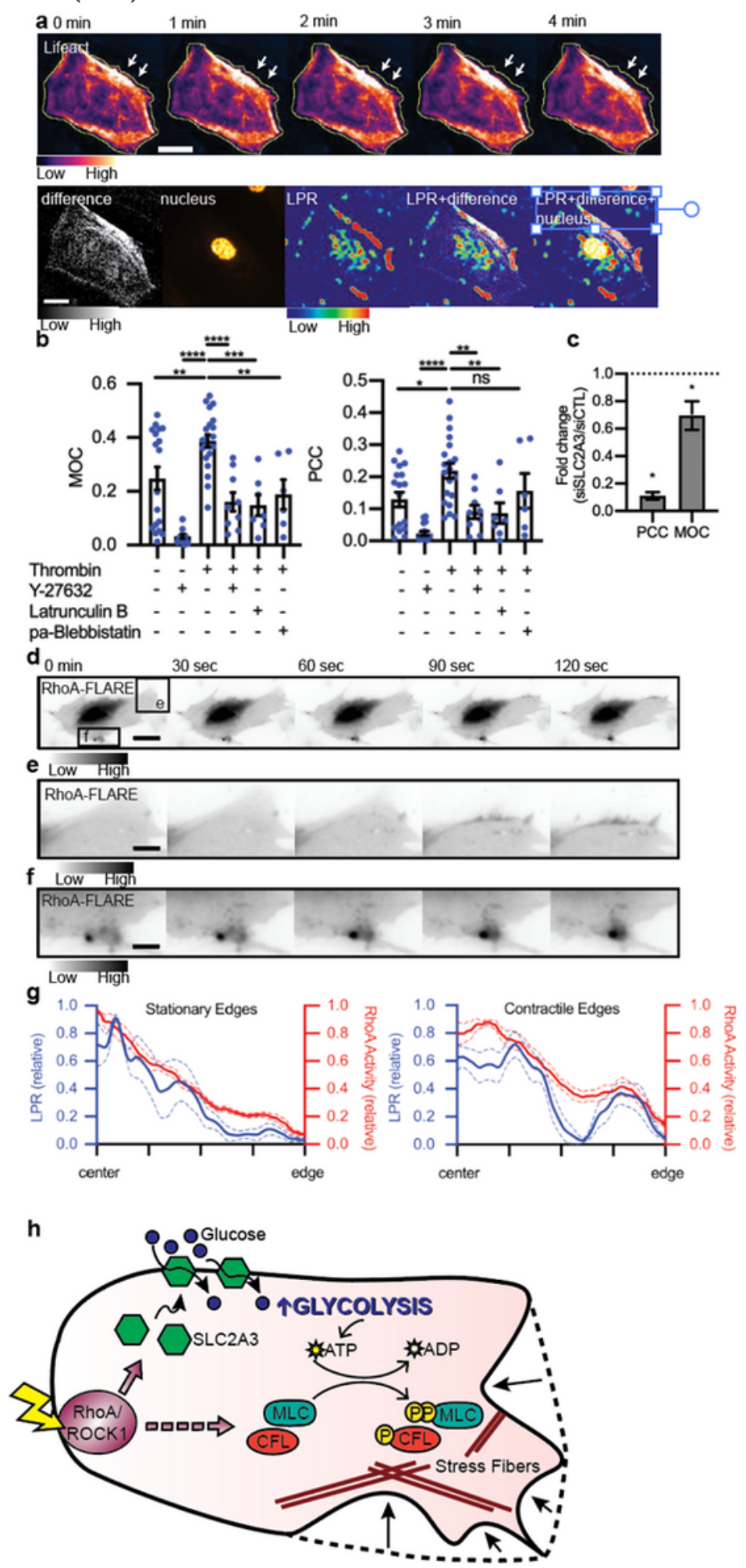

Figure 6

Subcellular distribution of LPR colocalizes with actin remodeling. a, Representative Montage of endothelial cell actin structure, as labeled with Lifeact-RFP, where the yellow line indicates original cell edge and white arrows point to contractile edge after treatment with thrombin. Maximum projection of 
change in actin structure (difference), nucleus, per pixel LPR map (LPR), and merges. Scale bar $=30 \square \mathrm{m}$. b, MOC and PCC of unstimulated or stimulated cells in the presence of ROCK inhibitor (Y-27632), actin polymerization inhibitor (Latrunculin B), and myosin II inhibitor (paBlebbistatin: para-aminoblebbistatin). ( $n=15,14,20,9,7,6$ from left to right). $c$, Fold change of PCC or MOC of thrombin treated cells under SLC2A3 knockdown compared to siCTRL. ( $n=9,17$ from left to right). $d$, RhoA activity measured by RhoA-FLARE in a contracting endothelial cell (scale bar $=15 \square \mathrm{m}$ ). e-f, Regions (e) and (f) from (d) demonstrate high RhoA activity in areas of active contraction (scale bar $=4.5 \square \mathrm{m}$ ). g, Normalized LPR and RhoA activity distribution from cell center to edge of non-contractile/stationary edges and contractile/motile edges stimulated with thrombin. (LPR stationary, $n=4$; RhoA stationary, $n=6$; LPR contract, $n=8$; RhoA contract, $n=9$ ). Dotted lines are standard deviation. $h$, Proposed mechanism of RhoA regulating glycolysis and contraction. Agonism of RhoA/ROCK1 stimulates SLC2A3 translocation to the membrane to increase intracellular glycolysis, thereby providing the substrate for phosphorylation of downstream targets (MLC and CFL), resulting in stress fiber formation and, ultimately, contraction. * $\mathrm{p}<$ $0.05,{ }^{*} \mathrm{p}<0.005, * \star * \mathrm{p}<0.0005, * \star \star \star \mathrm{p}<0.00005$. Statistical significance was determined by one-way ANOVA followed by Bonferroni test (b) or by Welch's t-test (c).

\section{Supplementary Files}

This is a list of supplementary files associated with this preprint. Click to download.

- SupplementalMethodsWuetalNATMETABL20032573.pdf

- ExtendedData.docx 